\title{
RESFRIAMENTO RÁPIDO E ARMAZENAMENTO DE CAQUIS (Diospyrus kaki, L.), CV. FUYU, EM CONDIÇÕES DE ATMOSFERA REFRIGERADA E MODIFICADA ${ }^{1}$
}

\author{
VALDECIR CARLOS FERRI ${ }^{2} \&$ CESAR VALMOR ROMBALDI ${ }^{3}$
}

\begin{abstract}
RESUMO - Para aumentar o período de conservação após a colheita de caquis, da cultivar Fuyu, dois sistemas de armazenamento foram estudados: atmosfera refrigerada (AR) e atmosfera modificada (AM). As frutas foram separadas em dois lotes: $1^{\circ}$ ) resfriamento rápido a $0^{\circ} \mathrm{C}$ até que a polpa atingisse $10^{\circ} \mathrm{C}$ e, então, armazenadas em $\mathrm{AR}$ a $10 \pm 0,5^{\circ} \mathrm{C}$ e $90 \pm 5 \% \mathrm{UR} ; 2^{\circ}$ ) resfriamento rápido a $0^{\circ} \mathrm{C}$ até que a polpa atingisse $0,5^{\circ} \mathrm{C}$, para serem armazenadas em condições de $\mathrm{AR}$ a $0 \pm 0,5^{\circ} \mathrm{C}$ e $90 \pm 5 \%$ de UR e em AM com filme PEBD $80 \mu \mathrm{m}$ a $0 \pm 0,5^{\circ} \mathrm{C}$ e $90 \pm 5 \%$ UR. Neste sistema (AM), também se testou o uso de sachet contendo $1 \mathrm{~g}$ de permanganato de potássio. Através dos resultados obtidos, observou-se que, para o armazenamento de caquis 'Fuyu', por períodos de até 30 dias, a temperatura de $10^{\circ} \mathrm{C}$ é eficiente e, para períodos superiores, o emprego de filmes de polietileno de baixa densidade de $80 \mu \mathrm{m}$, associado com a absorção do etileno, é o mais aficaz. O armazenamento em AR a $0 \pm 0,5^{\circ} \mathrm{Ce} 90 \pm 5 \%$ UR não foi eficiente na conservação dos caquis.
\end{abstract}

Termos para indexação: Diospyrus kaki, etileno, polietileno de baixa densidade,

\section{FAST COOLING AND STORAGE OF KAKIS (Diospyrus kaki, L.), CV. FUYU, IN CONDITIONS OF REFRIGERATED ATMOSPHERE AND MODIFIED}

\begin{abstract}
In order to increase the conservation period of kakis cultivar Fuyu after harvest, two storage systems were studied: refrigerated atmosphere (RA) and modified atmosphere (MA). The fruits were separated in two groups: 1 ) rapid cooling at $0^{\circ} \mathrm{C}$ until the pulp reached $10^{\circ} \mathrm{C}$ and, then stored in RA to $10 \pm 5^{\circ} \mathrm{C}$ and $90 \pm 5 \% \mathrm{RH} ; 2$ ) rapid cooling at $0{ }^{\circ} \mathrm{C}$ until the pulp reached $0,5^{\circ} \mathrm{C}$ and, then stored in $\mathrm{RA}$ to $0 \pm 0,5^{\circ} \mathrm{C}$ and $90 \pm 5 \% \mathrm{RH}$; and in MA with LDPE film $80 \mu \mathrm{m} 0 \pm 0.5^{\circ} \mathrm{C}$ and $90 \pm 5 \% \mathrm{RH}$. In the systems (MA), also the sachet containing $1 \mathrm{~g}$ of potassium permanganate were tested. Through the obtained results it was observed that for the storage of kakis 'Fuyu', for periods until 30 days, the temperature of $10{ }^{\circ} \mathrm{C}$ is efficient and, for longer periods the use of films of polyethylene of low density (LPDE) of $80 \mu \mathrm{m}$, associated with the absorption of the ethylene is also efficient. The storage in RA at $0 \pm 0,5^{\circ} \mathrm{C}$ and $90 \pm 5 \% \mathrm{RH}$ was not efficient in the conservation of kakis.
\end{abstract}

Index terms: Diospyrus kaki, ethylene, polyethylene of low density

\section{INTRODUÇÃO}

Os caquis são frutas climatéricas, com alta sensibilidade ao etileno e, se comparadas à maioria das frutas, podem ser classificadas como medianamente perecíveis (Brackmann et al., 1997). Para aumentar o período de conservação após a colheita, três sistemas de armazenamento têm sido preconizados: armazenamento em atmosfera refrigerada convencional (AR), armazenamento refrigerado em atmosfera modificada (AM) e armazenamento refrigerado em atmosfera controlada (AC) (Chitarra \& Chitarra, 1990; Ben-Arie \& Zutkhi, 1992).

A maioria dos trabalhos realizados (Chitarra \& Chitarra, 1990; Brackmann et al., 1999 e Rombaldi, 1999), visando à conservação de caquis em AR, recomendam temperaturas de armazenamento entre - 0,5 e $+0,5^{\circ} \mathrm{C}$ e umidade relativa entre 85 a $90 \%$. Neste sistema, dependendo da cultivar e das condições edafoclimáticas de cultivo, manejo e condições de colheita, o período de armazenamento seguro varia de 15 a 30 dias. Entretanto, estes trabalhos destacam a perda de qualidade das frutas, especialmente devido à deficiente evolução da coloração, ao aumento do escurecimento da epiderme e da degenerescência da polpa. Isto tem limitado, por exemplo, o transporte das frutas para regiões distantes e/ ou para a exportação. Além disso, a incidência de distúrbios aumenta com as oscilações na temperatura, fato comumente registrado no sistema de transporte rodoviário, aéreo e marítimo (Kluge et al., 1997).

Para melhorar o potencial de conservação, pode-se associar à AR, precedido de resfriamento rápido (Cortez et al., 2002), o emprego de filmes plásticos de Polietileno de Baixa Densidade (PEBD), com espessuras variando de 2 a $80 \mu \mathrm{m}$, gerando uma AM. Nestas condições, Rinaldi et al. (1998) verificaram que se pode duplicar o período seguro de estocagem em relação à $\mathrm{AR}$, reduzindo a perda de firmeza de polpa e a ocorrência de distúrbios fisiológicos. Entretanto, além da baixa operacionalidade e do custo da embalagem, há necessidade, para cada cultivar e região de origem, de calibrar-se o manejo do sistema. De maneira geral, para caquis da cultivar Fuyu produzidos na região Sul do Brasil, recomendam-se filmes de PEBD de 20 a $80 \mu \mathrm{m}$.

$\mathrm{O}$ uso de $\mathrm{AC}$ para caquis no Brasil ainda restringe-se a trabalhos de pesquisa, onde poucos trabalhos foram realizados (Brackmann et al., 1997; e Rinaldi et al.,1998). Os dados preliminares indicam que o sistema permite melhores condições de conservação do que a AM e a AR, prolongando em 20 e 60 dias, respectivamente, o período seguro de estocagem em relação a estes sistemas. Entretanto, é um sistema de maior custo de implantação e de menor versatilidade, já que o enchimento e o esvaziamento da câmara deverão ser realizados, cada um, em operação única. Além disto, se o sistema for utilizado unicamente para caquis, resultará numa ociosidade de mais de 8 meses por ano.

Por tratar-se de uma fruta sensível à ação do etileno, o emprego de sistemas que diminuam a produção e/ou a ação do etileno, pode contribuir para o prolongamento da vida útil dos caquis. Como concentrações acima de $0,01 \mathrm{ppm}$ já induzem uma forte aceleração do metabolismo de caquis, induzindo a maturação e a senescência, tornase mais interessante agir no controle da ação do que na produção. Por isso, tem sido recomendado o emprego de sistemas de absorção/ eliminação de etileno e/ou sistemas de inibição da ação, tais como os desenvolvidos com kiwi (Ferri et al., 1996).

Baseando-se nestas considerações, estudaram-se as combinações de: dois ajustes de temperaturas de polpa em tempos de resfriamento rápido, com a finalidade de obter a melhor retirada do calor de campo dos caquis da cultivar Fuyu; com armazenamentos em sistemas refrigerado convencional ou modificado com filmes de polietileno, visando à conservação das frutas, por um período seguro mínimo de 30 dias; o que garantiria à qualidade do produto para comercialização em outras regiões e/ou até mesmo em outros países.

\section{MATERIAL E MÉTODOS}

O experimento foi realizado com caquis, cultivar Fuyu, colhidos na safra 1999-2000, na propriedade de Nelson Valandro, de Farroupilha-

\footnotetext{
${ }^{1}$ (Trabalho 087/2003). Recebido: 07/07/2003. Aceito para publicação: 23/03/2004.

${ }^{2}$ Eng $^{\circ}$. Agr ${ }^{\circ}$., Dr., Professor ProDoc do Departamento de Ciência e Tecnologia Agroindustrial da FAEM/UFPel. Cx. Postal 354, CEP 90.100-900, PelotasRS. Telefone (53) 2757258. E-mail: leferri@ufpel.thce.br

${ }^{3}$ Eng $^{\circ}$. Agr ${ }^{\circ}$., Dr., Professor adjunto do Departamento de Ciência e Tecnologia Agroindustrial da FAEM/UFPel.
} 
RS. A colheita foi realizada quando as frutas apresentavam coloração amarelo-alaranjada, $70 \mathrm{~N}$ de firmeza de polpa, $15,6^{\circ} \mathrm{Brix}$ de sólidos solúveis totais (SST), 0,4 cmol. $\mathrm{L}^{-1}$ de acidez total titulável (ATT), 0,12mg. $\mathrm{kg}^{-1} \mathrm{de}$ clorofilas e $0,26 \mathrm{mg} \cdot \mathrm{kg}^{-1}$ de carotenóides.

Após a colheita, as frutas foram separadas em dois lotes: um, onde os caquis foram resfriados rapidamente em câmara frigorífica a $0 \pm 0,5^{\circ} \mathrm{C}$, até que a temperatura da polpa atingisse $10^{\circ} \mathrm{C}$ (4 horas), seguindose o armazenamento em Atmosfera Refrigerada (AR) a $10^{\circ} \mathrm{C} \mathrm{e} 90 \pm 5 \%$ de umidade relativa. No segundo lote, os caquis foram resfriados rapidamente em câmara frigorífica a $0 \pm 0,5^{\circ} \mathrm{C}$, até que a temperatura da polpa atingisse $0 \pm 0,5^{\circ} \mathrm{C}$ ( 9 horas). Completado o resfriamento rápido, as frutas foram armazenadas em $\mathrm{AR}$ a $0 \pm 0,5^{\circ} \mathrm{C}$ e $90 \pm 5 \%$ de umidade relativa, Atmosfera Modificada (AM) com filme de PEBD com espessura de $80 \mu \mathrm{m}$ e dimensões de $48 \times 26 \mathrm{~cm}$ selados a quente, a $0 \pm 0,5^{\circ} \mathrm{C}$ e $90 \pm 5 \%$ de umidade relativa; e AM com filme de PEBD com espessura de $80 \mu \mathrm{m}$ e dimensões de $48 \times 26 \mathrm{~cm}$ selados a quente, a $0 \pm 0,5^{\circ} \mathrm{C}$ e $90 \pm 5 \%$ de umidade relativa, associada ao uso de sachet de $1 \mathrm{~g}$ de permanganato de potássio homogeneizado a $2 \mathrm{~g}$ de vermiculita de granulação fina. As unidades experimentais foram armazenadas por 30 ou 60 dias, visando a garantias da qualidade de caquis comercializados em outras regiões e/ou países.

$\mathrm{O}$ delineamento experimental adotado foi o inteiramente casualizado. Cada unidade experimental foi representada por 12 frutas, tanto em AR como nos filmes que compunham as embalagens de AM, em quatro repetições.

Para a avaliação da qualidade das frutas, determinaram-se, na instalação do experimento, aos 30 e 60 dias de armazenamento, a firmeza de polpa (FP), a acidez total titulável (ATT), o teor de sólidos solúveis totais (SST), o conteúdo de clorofilas e carotenóides, a coloração, a ocorrência de escurecimento da epiderme e de degenerescência interna e a concentração de etileno no interior das embalagens. A primeira análise foi realizada 12 horas após a retirada das frutas da câmara frigorífica e a segunda, 6 dias após. Neste último caso, buscou-se simular as condições de comercialização, mantendo as amostras a $23 \pm 3^{\circ} \mathrm{C}$ e $75 \pm 5 \%$ de umidade relativa.

Para análise, utilizaram-se os parâmetros - i) firmeza de polpa: determinada com auxílio de penetrômetro manual, com ponteira de $8 \mathrm{~mm}$ de diâmetro, com resultados expressos em Newton (N); ii) teor de SST: obtido através de refratometria, com resultados expressos em ${ }^{\circ}$ Brix; iii) a ATT: determinada por titulação química de neutralização, com fenolfetaleína a $0,25 \%$ como indicador de final de reação, em adição de hidróxido de sódio $(\mathrm{NaOH}) 0,1 \mathrm{~N}$ até a viragem em $\mathrm{pH} 8,2$. Os resultados foram expressos em cmol. $\mathrm{L}^{-1}$; iv) para coloração, adotou-se uma escala subjetiva com as seguintes atribuições de notas às frutas: 1-verdeamarelada; 2-amarelo-alaranjada; 3-alaranjada; 4-vermelha, e 5-vermelhointensa; v) conteúdo de clorofilas e de carotenóides foram determinados através da metodologia descrita em Lutz (1985), com resultados expressos em mg. $\mathrm{kg}^{-1}$ de peso fresco das frutas; vi) produção de etileno: determinado por cromatografia em fase gasosa, expressa em $\mathrm{nl} \cdot \mathrm{h}^{-1} \cdot \mathrm{g}^{-1}$, utilizando-se de um cromatógrafo a gás, marca Varian ${ }^{\circledR}$, modelo 8000 , equipado com uma coluna de aço inox 1/8", preparado com Porapak ${ }^{\circledR} \mathrm{N}$, e um detector de ionização de chama. As temperaturas da câmara de injeção, da coluna e do detector foram de $80^{\circ} \mathrm{C}, 90^{\circ} \mathrm{C}$ e $200^{\circ} \mathrm{C}$, respectivamente. Utilizou-se, como padrão, uma solução de etileno a 10ppm. Para a determinação da produção de etileno, dois frutos foram condicionados em frascos hermeticamente fechados, durante uma hora, a $25^{\circ} \mathrm{C}$. Passado esse período, coletou-se, com auxílio de seringas hipodérmicas, $1 \mathrm{~mL}$ da atmosfera gasosa; vii) degenerescência interna: foi considerada quando mais do que $50 \%$ da polpa se apresentava translúcida; o escurecimento da epiderme: foram consideradas frutas com escurecimento da epiderme quando mais do que $20 \%$ da superfície se apresentavam com o sintoma. Os resultados foram expressos em percentagem.

Para a comparação de médias dos tratamentos, os resultados das variáveis, no ato de instalação e aos 30 e 60 dias, foram submetidos à análise de variância, utilizando-se do teste de Duncan, a 5\% de probabilidade, exceto para degenerescência interna e coloração, que foram analisadas por regressão polinomial.

\section{RESULTADOSEDISCUSSÃO}

Comparando-se o efeito das condições de armazenamento, após 30 dias de estocagem e 12 horas em temperatura ambiente, pôde-se verificar que todas as frutas se apresentaram em condições de comercialização (Tabela 1). Entretanto, o armazenamento em AM proporcionou maior manutenção da firmeza de polpa, com valores médios de $67 \mathrm{~N}$, enquanto em $\mathrm{AR}$ a $0^{\circ} \mathrm{C}$ e a $10^{\circ} \mathrm{C}$, foram de $59 \mathrm{~N}$ e $50 \mathrm{~N}$, respectivamente.

TABELA 1 - Características de caquis da cultivar Fuyu armazenados por 30 dias em atmosferas refrigerada convencional (AR) a $0^{\circ} \mathrm{C}$ e $10^{\circ} \mathrm{C}$ e modificada (AM), com e sem absorção de etileno, e mantidos em temperatura ambiente por 12 horas.

\begin{tabular}{llllc}
\hline Parâmetros & \multicolumn{4}{c}{ Sistemas de armazenamento } \\
\cline { 2 - 6 } & $0^{\circ} \mathrm{C}$ & $10^{\circ} \mathrm{C}$ & $\mathrm{AM}$ & $\mathrm{AM}+\mathrm{Abs}$ \\
\hline Firmeza de polpa $(\mathrm{N})$ & $59 \mathrm{~b}$ & $50 \mathrm{c}$ & $67 \mathrm{a}$ & $67 \mathrm{a}$ \\
SST $\left({ }^{0}\right.$ Brix) & $15.8 \mathrm{ab}$ & $16,1 \mathrm{a}$ & $15,6 \mathrm{~b}$ & $15,7 \mathrm{~b}$ \\
ATT (cmol. L-1) & $0.6 \mathrm{a}$ & $0.5 \mathrm{ab}$ & $0.4 \mathrm{~b}$ & $0.4 \mathrm{~b}$ \\
Coloração $(1-5)$ & $2 \mathrm{~b}$ & $4 \mathrm{a}$ & $2 \mathrm{~b}$ & $2 \mathrm{~b}$ \\
Clorofila $(\mu \mathrm{g} / \mathrm{mg})$ & $0.10 \mathrm{a}$ & $0.02 \mathrm{~b}$ & $0.11 \mathrm{a}$ & $0.12 \mathrm{a}$ \\
Carotenóides $(\mu \mathrm{g} / \mathrm{mg})$ & $0.25 \mathrm{~b}$ & $0.33 \mathrm{a}$ & $0.26 \mathrm{~b}$ & $0.25 \mathrm{~b}$ \\
Etileno $(\mathrm{ppm})$ & $\mathrm{Nd}$ & $\mathrm{Nd}$ & $13 \mathrm{a}$ & $0.02 \mathrm{~b}$ \\
Degenerescência da polpa $(\%)$ & 00 & 30 & 00 & 00 \\
Escurecimento da epiderme $(\%)$ & 00 & 00 & 00 & 00
\end{tabular}

* Médias seguidas por letras distintas, nas linhas, diferem entre si, pelo teste de Duncan, ao nível de 5\% de significância.

$\mathrm{Nd}=$ dados não detectados

Outra variação, facilmente perceptível entre os tratamentos, foi a evolução da coloração das frutas armazenadas a $10^{\circ} \mathrm{C}$. Os caquis colhidos com coloração amarelo-alaranjada (valor 2 na escala) evoluíram para o vermelho (valor 4). Já, nos demais tratamentos, não houve evolução da coloração. Esta diferença é comercialmente importante, já que a coloração atrativa constitui-se num dos principais atributos de qualidade de caquis.

Outros autores (Brackmann et al., 1997; Rinaldi, 1998 e Rinaldi et al., 1998) também observaram que caquis armazenados sob refrigeração, com temperatura abaixo de $5^{\circ} \mathrm{C}$, não apresentam uma significativa evolução da coloração. Porém, as causas bioquímico-moleculares ainda não foram elucidadas. Pelos resultados apresentados na Tabela 1, pôdese verificar que, justamente nas frutas onde houve melhoria da coloração, houve redução significativa do conteúdo de clorofilas e os maiores incrementos de conteúdos de carotenóides. Baseando-se nestes resultados, acredita-se que as baixas temperaturas inibam as vias de biossíntese de pigmentos hidrofóbicos (carotenóides).

O emprego de produto absorvedor de etileno reduziu a concentração deste hormônio para teores médios de $0,02 \mathrm{ppm}$. Porém, este período de avaliação (30 dias + 12 horas) não se mostrou necessário para manter as frutas em condições para a comercialização.

A manutenção das frutas em condições de comercialização simulada, durante 6 dias (Tabela 2), ampliou as diferenças entre os tratamentos. Mais especificamente, destacam-se as reduções de firmeza de polpa, incremento de coloração e de degenerescência da polpa.

Após 30 dias de armazenamento refrigerado e 6 dias em condições de comercialização simulada, os caquis armazenados a $0^{\circ} \mathrm{C}$ apresentaram perda total da firmeza de polpa, enquanto aqueles armazenados a $10^{\circ} \mathrm{C}$ se mantiveram com valores médios de $34 \mathrm{~N}$ e coloração avermelhada. Além disto, em caquis armazenados a $0^{\circ} \mathrm{C}$, houve incidência de degenerescência da polpa em $100 \%$ das frutas, sem evolução da coloração. Quando o armazenamento foi conduzido em atmosfera modificada, a firmeza de polpa foi significativamente superior e houve efeito positivo da absorção do etileno. Também se registrou melhora na coloração, passando de amarelo-alaranjada (2) para alaranjada (3). 
TABELA 2 - Características de caquis da cultivar Fuyu armazenados por 30 dias em atmosferas refrigerada convencional (AR) a $0^{\circ}$ $\mathrm{Ce} 10^{\circ} \mathrm{C}$ e modificada (AM), com e sem absorção de etileno, e mantidos em temperatura ambiente por 6 dias.

\begin{tabular}{llllc}
\hline Parâmetros & \multicolumn{4}{c}{ Sistemas de armazenamento } \\
\cline { 2 - 5 } & $0^{\circ} \mathrm{C}$ & $10^{\circ} \mathrm{C}$ & $\mathrm{AM}$ & $\mathrm{AM}+\mathrm{Abs}$. \\
\hline Firmeza de polpa (N) & $09 \mathrm{~d}$ & $34 \mathrm{c}$ & $58 \mathrm{~b}$ & $64 \mathrm{a}$ \\
$\mathrm{SST}\left({ }^{0}\right.$ Brix) & 15.5 & 16.2 & 16.2 & 16.0 \\
ATT $(\mathrm{cmol} . \mathrm{L}-1)$ & $0.72 \mathrm{a}$ & $0.52 \mathrm{~b}$ & $0.48 \mathrm{~b}$ & $0.47 \mathrm{~b}$ \\
Coloração $(1-5)$ & $2 \mathrm{c}$ & $5 \mathrm{a}$ & $3 \mathrm{~b}$ & $3 \mathrm{~b}$ \\
Clorofila $(\mu \mathrm{g} / \mathrm{mg})$ & $0.12 \mathrm{a}$ & $0.02 \mathrm{~b}$ & $0.10 \mathrm{a}$ & $0.11 \mathrm{a}$ \\
Carotenóides $(\mu \mathrm{g} / \mathrm{mg})$ & $0.28 \mathrm{~b}$ & $0.45 \mathrm{a}$ & $0.28 \mathrm{~b}$ & $0.30 \mathrm{~b}$ \\
Etileno $(\mathrm{ppm})$ & $\mathrm{Nd}$ & $\mathrm{Nd}$ & $\mathrm{Nd}$ & $\mathrm{Nd}$ \\
Degenerescência da polpa $(\%)$ & $100 \mathrm{a}$ & $30 \mathrm{~b}$ & $00 \mathrm{c}$ & $00 \mathrm{c}$ \\
Escurecimento da epiderme $(\%)$ & 00 & 00 & 00 & 00
\end{tabular}

* Médias seguidas por letras distintas, nas linhas, diferem entre si, pelo teste de Duncan, ao nível de $5 \%$ de significância.

$\mathrm{Nd}=$ dados não detectados.

O conjunto destes resultados indica que, para situações de programação de armazenamento por períodos de até 30 dias, e de mais 6 dias para a comercialização em condições ambientais, não há necessidade de utilização do sistema de AM, nem absorção de etileno. Da mesma forma que, também, o armazenamento a $0^{\circ} \mathrm{C}$ não foi eficiente, já que há significativa perda de firmeza de polpa e elevada ocorrência de distúrbios fisiológicos, sem melhoria da coloração. No armazenamento a $10^{\circ} \mathrm{C}$, além de as frutas apresentaram-se com melhor aparência e com firmeza de polpa acima do mínimo necessário para a comercialização/consumo, que é de $20 \mathrm{~N}$, representa menor custo operacional e energético do sistema (Mitcham et al., 1998).

Quando se avaliaram as frutas após 60 dias de armazenamento (Tabela 3), verificou-se que aquelas armazenadas a $10^{\circ} \mathrm{C}$ apresentavam a menor firmeza de polpa, com valores médios de $10 \mathrm{~N}$. Nos demais sistemas, a firmeza de polpa situou-se acima de 30N. Os melhores resultados foram obtidos nos caquis armazenados em AM com absorção do etileno, onde se obtiveram $63 \mathrm{~N}$.

TABELA 3 - Características de caquis da cultivar Fuyu armazenados por 60 dias em atmosferas refrigerada convencional (AR) a $0^{\circ}$ $\mathrm{Ce} 10^{\circ} \mathrm{C}$ e modificada (AM), com e sem absorção de etileno, e mantidos em temperatura ambiente por 12 horas.

\begin{tabular}{llllc}
\hline Parâmetros & \multicolumn{4}{c}{ Sistemas de armazenamento } \\
\cline { 2 - 5 } & $0^{\circ} \mathrm{C}$ & $10^{\circ} \mathrm{C}$ & $\mathrm{AM}$ & $\mathrm{AM}+\mathrm{Abs}$ \\
\hline Firmeza de polpa $(\mathrm{N})$ & $33 \mathrm{c}$ & $10 \mathrm{~d}$ & $56 \mathrm{~b}$ & $63 \mathrm{a}$ \\
SST $\left({ }^{0}\right.$ Brix) & $15.6 \mathrm{a}$ & $14.8 \mathrm{~b}$ & $15.5 \mathrm{a}$ & $15.6 \mathrm{a}$ \\
ATT $(\mathrm{cmol}$. L-1) & $0.6 \mathrm{a}$ & $0.5 \mathrm{a}$ & $0.5 \mathrm{a}$ & $0.5 \mathrm{a}$ \\
Coloração $(1-5)$ & $2 \mathrm{c}$ & $5 \mathrm{a}$ & $3 \mathrm{~b}$ & $3 \mathrm{~b}$ \\
Clorofila $(\mu \mathrm{g} / \mathrm{mg})$ & $0.112 \mathrm{a}$ & $0.02 \mathrm{~b}$ & $0.11 \mathrm{a}$ & $0.11 \mathrm{a}$ \\
Carotenóides $(\mu \mathrm{g} / \mathrm{mg})$ & $0.25 \mathrm{c}$ & $0.36 \mathrm{a}$ & $0.30 \mathrm{~b}$ & $0.30 \mathrm{~b}$ \\
Etileno (ppm) & $\mathrm{Nd}$ & $\mathrm{Nd}$ & $18 \mathrm{a}$ & $0.1 \mathrm{~b}$ \\
Degenerescência da polpa $(\%)$ & $100 \mathrm{a}$ & $100 \mathrm{a}$ & $00 \mathrm{c}$ & $00 \mathrm{c}$ \\
Escurecimento da epiderme $(\%)$ & $30 \mathrm{~b}$ & $45 \mathrm{a}$ & $20 \mathrm{c}$ & $00 \mathrm{~d}$ \\
\hline
\end{tabular}

* Médias seguidas por letras distintas, nas linhas, diferem entre si, pelo teste de Duncan, ao nível de $5 \%$ de significância.

$\mathrm{Nd}=$ dados não detectados.

À semelhança do que foi observado aos 30 dias (Tabelas 1 e 2), a melhor coloração foi observada em caquis armazenados a $10^{\circ} \mathrm{C}(5)$ e em $\operatorname{AM}(3)$.

No que tange aos distúrbios fisiológicos, observou-se uma alta incidência de degenerescência em frutas armazenadas a $10^{\circ} \mathrm{Ce} 0^{\circ} \mathrm{C}$, atingindo $100 \%$ dos caquis. Também detectou a ocorrência de escurecimento da epiderme, atingindo $30 \%$ das frutas armazenadas a $0^{\circ}$ C, $45 \%$ das armazenadas a $10^{\circ} \mathrm{Ce} 20 \%$ das armazenadas em AM.

A absorção do etileno proporcionou, além da maior preservação da firmeza de polpa, uma proteção à ocorrência de distúrbios fisiológicos. Pech et al. (1994) citam que a redução da ação do etileno pode reduzir a velocidade e a intensidade de ocorrência de distúrbios fisiológicos, incluindo os danos pelo frio. Brackmann et al. (1999), também observaram redução no escurecimento da epiderme com a utilização de absorvedores de etileno.

A manutenção dos caquis, após 60 dias de armazenamento, por 6 dias em condições de comercialização simulada (Tabela 4), intensificou as diferenças observadas anteriormente, e somente as frutas armazenadas em AM mantiveram condições mínimas para a comercialização. Porém, destacou-se o efeito da absorção de etileno, que proporcionou melhores resultados, controlando a ocorrência de escurecimento da epiderme.

TABELA 4 - Características de caquis da cultivar Fuyu armazenados por 60 dias em atmosferas refrigerada convencional (AR) a $0^{\circ}$ $\mathrm{Ce} 10^{\circ} \mathrm{C}$ e modificada (AM), com e sem absorção de etileno, e mantidos em temperatura ambiente por 6 dias.

\begin{tabular}{lllll}
\hline Parâmetros & \multicolumn{4}{c}{ Sistemas de armazenamento } \\
\cline { 2 - 5 } & $0^{\circ} \mathrm{C}$ & $10^{\circ} \mathrm{C}$ & $\mathrm{AM}$ & $\mathrm{AM}+\mathrm{Abs}$ \\
\hline Firmeza de polpa (N) & $00 \mathrm{~d}$ & $2.0 \mathrm{c}$ & $43 \mathrm{~b}$ & $49 \mathrm{a}$ \\
SST $\left({ }^{0}\right.$ Brix) & $15.8 \mathrm{a}$ & $14.9 \mathrm{~b}$ & $15.7 \mathrm{a}$ & $15.6 \mathrm{a}$ \\
ATT (cmol. L -1) & $0.8 \mathrm{a}$ & $0.5 \mathrm{c}$ & $0.6 \mathrm{~b}$ & $0.6 \mathrm{~b}$ \\
Coloração $(1-5)$ & $2 \mathrm{c}$ & $5 \mathrm{a}$ & $3 \mathrm{~b}$ & $3 \mathrm{~b}$ \\
Clorofila $(\mu \mathrm{g} / \mathrm{mg})$ & $0.10 \mathrm{a}$ & $0.02 \mathrm{~b}$ & $0.10 \mathrm{a}$ & $0.10 \mathrm{a}$ \\
Carotenóides $(\mu \mathrm{g} / \mathrm{mg})$ & $0.28 \mathrm{c}$ & $0.46 \mathrm{a}$ & $0.36 \mathrm{~b}$ & $0.36 \mathrm{~b}$ \\
Etileno $(\mathrm{ppm})$ & $\mathrm{Nd}$ & $\mathrm{Nd}$ & $\mathrm{Nd}$ & $\mathrm{Nd}$ \\
Degenerescência da polpa $(\%)$ & $100 \mathrm{a}$ & $100 \mathrm{a}$ & $00 \mathrm{~b}$ & $00 \mathrm{~b}$ \\
Escurecimento da epiderme $(\%)$ & $50 \mathrm{a}$ & $50 \mathrm{a}$ & $30 \mathrm{~b}$ & $00 \mathrm{c}$ \\
\hline
\end{tabular}

* Médias seguidas por letras distintas, nas linhas, diferem entre si, pelo teste de Duncan, ao nível de 5\% de significância.

$\mathrm{Nd}=$ dados não detectados

\section{CONCLUSÕES}

$\mathrm{O}$ armazenamento em $\mathrm{AR}$ a $10^{\circ} \mathrm{Ce} 90 \pm 5 \%$ de umidade relativa, precedido de resfriamento rápido a $0 \pm 0,5^{\circ} \mathrm{C}$ (temperatura da polpa de $10^{\circ} \mathrm{C}$ ), é eficiente no controle da maturação de caquis, da cultivar Fuyu, por período de 30 dias; já a AM com filme de PEBD ( $80 \mu \mathrm{m}, 48 \times 26 \mathrm{~cm})$, a $0 \pm 0,5^{\circ} \mathrm{C}$ e $90 \pm 5 \%$ de umidade relativa, associada a sachet de permanganato de potássio $(1 \mathrm{~g} / 12$ frutas $)$ preserva os caquis em condições adequadas de consumo por 60 dias.

\section{REFERÊNCIAS BIBLIOGRÁFICAS}

BEN-ARIE, R.; ZUTKHI, Y. Extending the storage life of "Fuyu" persimmon by modified-atmosphere packaging. HortScience, Alexandria, v.27, n.7, p.811-813.1992

BRACKMANN, A.; MAZARO, S.M.; SAQUET, A.A.. Frigoconservação de caquis (Diospyros kaki, L.) das cultivares Fuyu e Rama Forte. Ciência Rural, Santa Maria, v.27, n.4, p.561-565. 1997.

BRACKMANN, A.; STEFFENS, C.A.; MAZARO, S.M. Armazenamneto de caquis (Diospyros kaki, L.), cv. Fuyu, em condições de atmosfera modificada e controlada. Revista Brasileira de Armazenamento, Viçosa, v.24, n.2, p.42-46. 1999.

CHITARRA, A. B.; CHITARRA, M.I.F. Pós-colheita de frutos e hortaliças: fisiologia e manuseio. Lavras: ESAL/FAEPE, 1990. 293p.

CORTEZ,L.A.B.; HONÓRIO, S.L.; MORETTI, C.L. Resfriamentode frutas e hortaliças. Embrapa Hortaliças: Brasília, 2002. 428p.

FERRI, V.C.; BILHALVA, A.B.; CASAGRANDE Jr, J.G. 1996. Efeito do permanganato de potássio na conservação de futos de kiwi (Actinidia deliciosa, Lid.) cultivar Monty. In: CONGRESSO BRASILEIRODE FRUTICULTURA, 14.,1996, Curitiba. Anais... p.16.

KLUGE, R.A.; NACHTIGAL, J.C.; FACHINELLO, J.C.; BILHALVA,A.B. Fisiologia e manejo pós-colheita de frutas de clima temperado. Pelotas: UFPel, 1997. 
LUTZ, A. Normas analíticas do Instituto Adolf Lutz: métodos químicos para análise de alimentos. 3. ed. São Paulo, 1985. 533p.

MITCHAM, J.E.; CRISOSTO, C.H.; KADER, A.A. Recommendations for maintaining postharvest quality. Davis:Department of Pomology, University of California, Davis, 1998. 120p.

PECH, J.C.; LATCHÉ, A.; BALAGUÉ, C.; BOUZAYEN, M.; LELIÈVRE, J.M. Postharvest physiology of climateric fruits: recent development in the biosyntesis and action of ethylene. Sciencia Alim., v.14, p.314, 1994.

RINALDI, M.M.; FERRI, V.C.; ROMBALDI, C.V. 1998. Frigoconservação de caquis (Diospyros kaki, L.) cv. Fuyu, em atmosfera modificada.
In: CONGRESSO BRASILEIRO DECIÊNCIA E TECNOLOGIADE ALIMENTOS, 16., 1998, Rio de Janeiro, RJ. Anais... Rio de Janeiro: S.B.C.T.A., Universidade Federal do Rio de Janeiro. 1998, v.2, p.415420.

RINALDI, M.M. Frigoconservação de caquis (Diospyros kaki, L.) cv. Fuyu, em atmosfera modificada. 1998. 22f. Dissertação (Mestrado em Ciência e Tecnologia Agroindustrial) - Universidade Federal de Pelotas, Pelotas, 1998.

ROMBALDI, C. V. Armazenamento de caqui. Jornal da Fruta, Lages,n.232, p.4, 1999. 